\title{
Effects of irrelevant level fluctuation on frequency discrimination by young and older adults
}

\author{
Blas Espinoza-Varas ${ }^{1}$ and Hyunsook Jang ${ }^{2}$ \\ ${ }^{1}$ Department of Communication Sciences and Disorders, University Oklahoma Health Sciences Center, \\ Oklahoma City, Oklahoma 73104, USA \\ ${ }^{2}$ Division of Speech Pathology and Audiology, Hallym University, \\ Chuncheon, South Korea
}

(Received 22 February 2005, Accepted for publication 22 March 2005)

Keywords: Frequency discrimination, Level fluctuation, Aging, Attention, Memory PACS number: 43.66.Fe, 43.66.Sr [DOI: 10.1250/ast.26.394]

\section{Introduction}

The present experiments assessed effects of irrelevant fluctuation in signal level on frequency discrimination thresholds (FDTs) of young and older adults; more generally, age effects on the ability to ignore irrelevant auditory information were studied in psychoacoustic discrimination tasks. Previous research suggests that this ability degrades with aging, and that performance decrements induced by irrelevant auditory information are more severe in older than in young adults [1-3]. However, most of the evidence comes from studies using speech-recognition tasks; frequency discrimination tasks with simple sinusoids have not been employed thus far.

In order to convey irrelevant information, the auditory display must include two or more sources of discernible information. In addition, the sources must provide conflicting information so that, to respond correctly, subjects must succeed both at attending to one (relevant) source and at ignoring the other (irrelevant) sources. That is, subjects must be able to extricate relevant and irrelevant information at the perceptual level. The mere addition of noise, stimulus context or variability does not necessarily create irrelevant information; to do so, systematic stimulus differences that are discernible yet inconsistent with relevant information must be displayed also [4,5].

In two-interval, two-alternative, forced-choice (2IAFC) frequency discrimination tasks, irrelevant information can be engendered by fluctuation in the signal level, either within trials, between trials, or both [6,7]. Previous research shows that such random fluctuation has only small effects in the FDTs of well-trained young adults; compared to the fixed level condition, Weber fractions increase by a factor of 2.7 on average. That is, young adults have little difficulty ignoring random irrelevant level fluctuation in 2IAFC frequencydiscrimination tasks.

In the present study, a modification of the above paradigm was used to compare the ability to ignore irrelevant auditory information of young and older adults; in both groups, FDTs were measured with and without fluctuation in signal level. The effects of level-fluctuation polarity (positive or negative) and training were also studied. Because target information always consisted of positive frequency increments, it was assumed that the positive irrelevant level differences would be harder to ignore than the negative, owing to similarity and contrast effects. These effects would be of significance if the ignoring of irrelevant information were dependent on attention-like filtering mechanisms tuned to parameters of the target increment. On the other hand, irrelevant level differences could be difficult to ignore because they increase the stimulus set size, and thereby the amount of information and memory-capacity load $[1,8]$. If so, age effects on the ability to ignore irrelevant differences could be accounted for in terms of memory-capacity constraints; however, in contrast to the attention-filtering hypothesis, the predicted effects are not dependent on polarity. Finally, training effects were studied to determine if the ability to ignore irrelevant information depends on how familiar subjects are with the stimuli and task.

\section{Method}

\subsection{Experimental task}

The experiments measured frequency discrimination thresholds (FDTs) for 1,500-Hz, 40-ms, 70-80 dB SPL targets tones $(T)$ presented in quiet. In the three-interval, twoalternative, forced-choice (3I/2AFC) task, each trial presented a "standard" followed by two "comparisons," respectively, in the 1st, 2nd, and 3rd observation interval, with 500-ms of silence between intervals (see Fig. 1). A positive frequency increment was added to one comparison, selected randomly with equal probability, and subjects had to decide whether the increment was in the 2nd or 3rd interval; this is the baseline condition without irrelevant level fluctuation.

In level-fluctuation conditions, a positive target frequency increment was added again to one comparison, but both comparisons conveyed also positive or negative level differences. Figure 1 depicts a trial with 40-ms targets having simultaneous increments in frequency and irrelevant differences or fluctuation in level. In both comparisons there are positive irrelevant level differences $(\Delta L)$, but only in the first there is a target frequency increment $(\Delta F)$. In terms of level, both comparisons are different from the standard, but in terms of frequency, only one comparison is different from standard. To respond correctly, listeners had to attend to the target frequency increment while attempting to ignore the irrelevant level differences $[9,10]$.

The irrelevant level differences were \pm 5 , or $\pm 10 \mathrm{~dB}$ and the resulting loudness changes were easily noticed by all 


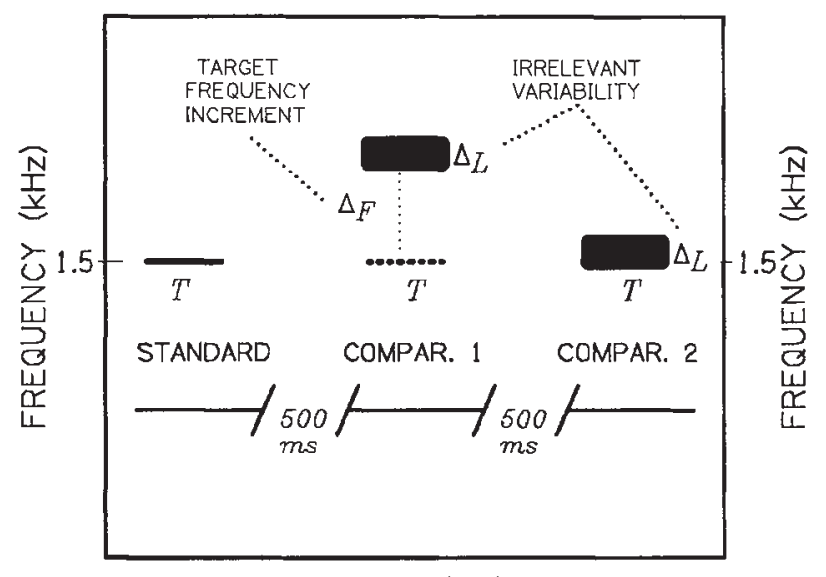

TIME (ms)

Fig. 1 Trial structure of the three-interval, two-alternative, forced-choice discrimination task. Each trial displayed a "standard" followed by two "comparison" target tones, respectively, in the 1st, 2nd and 3rd observation interval. In this depiction, comparison 1 conveys a target frequency increment $(\Delta F)$, represented by the elevation of the target along the ordinate. Comparisons 1 and 2 convey irrelevant differences in $T$ level $(\Delta L)$, represented by heavy bars. The listeners had to decide in which comparison was the target frequency higher than that of the standard, while attempting to ignore the irrelevant level differences.

normal-hearing subjects. In all cases, the size and polarity of the irrelevant level difference were the same for the two comparisons of a given trial. Within each trial block, polarity was held constant but the irrelevant-difference size (5 or $10 \mathrm{~dB}$ ) was sampled randomly with equal probability. Baseline conditions without irrelevant level differences were studied also.

\subsection{Threshold estimation}

The size of the target increment presented on a given trial was specified by an adaptive rule [11] that estimated frequency discrimination thresholds (FDTs) near the 71 percentcorrect level. FDT estimates were defined as the average of the frequency increments specified in the last six reversals of adaptive tracks comprising a total of eight reversals. FDT estimates from 5-6 adaptive tracks were averaged to obtain each FDT or data point [e.g., 12]. A minimum of 10 FDT estimates per experimental condition was collected on each subject. In all conditions, data collection terminated when: a) in a series of eight FDT estimates, subjects produced six FDTs approaching asymptotic values reported in the literature; or b) subjects completed 50 FDT estimates without meeting the previous criterion. Experimental sessions lasted $1-2 \mathrm{~h}$ and rest periods were allowed every $10-15 \mathrm{~min}$.

\subsection{Equipment}

Sinusoidal tones, gated with 4-ms rise-fall times, were synthesized (20,000 samples/s) with a signal-processing software package (LabVIEW 2) installed on a Macintosh IIfx computer. The analog signals coming out of the computer's 12-bit D/A converters were low-pass filtered at $4,500 \mathrm{~Hz}$, amplified, attenuated, and fed to earphones (Telephonics TDH-49). Stimuli were presented monaurally and calibrated with a Quest-155 SPL meter, a frequency counter, and an oscilloscope. Subjects worked at listening stations in a soundtreated room, and responded on a computer keyboard in accordance with instructions and feedback displayed on a CRT monitor. The LabVIEW software implemented the increments in frequency, and a programmable attenuator (TDT-PA1) implemented the differences in level. The Macintosh computer controlled all experimental events, including stimulus presentation, response recording, timing, and feedback.

2.4. Subjects

Six 65-74 years old (mean $=67$ ) subjects, in good health, comprised the older-adult group (3 were in the active workforce and 3 on retirement). Six graduate students, 21-39 years old (mean $=24.5$ ) enrolled at a metropolitan university health center comprised the young-adult group. In the audiometric frequencies, all six young and four older adults had hearing thresholds within the normal range. The other two elders' hearing thresholds were within the normal range in the $0.25-$ $4.0 \mathrm{kHz}$ frequencies, but outside the normal range above $4.0 \mathrm{kHz}$. Hearing thresholds did not exceed the ANSI-1996 standard [13] by more than $15 \mathrm{~dB}$ at the target signal frequency. In all subjects, the $1,500-\mathrm{Hz}$ target tone was clearly audible with a sensation level of 50-55 dB. All older adults scored above $90 \%$ correct in a mental-status questionnaire [14].

\section{Results}

All subjects were run in the baseline condition (without irrelevant level differences) until the FDTs approached asymptotic values reported in the literature [15]. This was followed by two conditions with either positive or negative irrelevant level differences. The test order of polarity conditions was randomized across trial blocks of each experimental session; within each trial block, all irrelevant differences had the same polarity.

Figure 2 depicts, as a function of training, the groupaverage FDTs measured in conditions having positive or negative irrelevant level differences; the baseline FDTs are shown also. In this Figure, the abscissa values represent the number of trial blocks completed by each group; each block yielded a single FDT estimate based on 14-54 trials $($ mean $=32)$. The amount of training increases directly with the number of trial blocks.

In the baseline condition, the older adults' FDTs were only slightly larger than the $2.0-2.5 \mathrm{~Hz} F D T s$ of young adults. In all subjects, the baseline FDTs were lower than $3.5 \mathrm{~Hz}$, in agreement with $F D T$ values reported earlier for frequencies higher than $1,000 \mathrm{~Hz}$ [e.g., 15]. Achieving these low FDTs required far more training in older than in young adults, however this procedure served the purpose of matching the baseline performance of the two age groups, prior to presenting irrelevant differences.

Regardless of polarity, irrelevant differences in target level failed to elevate the young adults' FDTs, and target frequency discrimination was very precise in all conditions, in close agreement with earlier studies [6]. A recent paper [16] did report significant FDT elevations, but the signal level fluctuation $(8-20 \mathrm{~dB})$ was much larger than the one used here $(5-10 \mathrm{~dB})$. At the beginning of training, positive and negative 


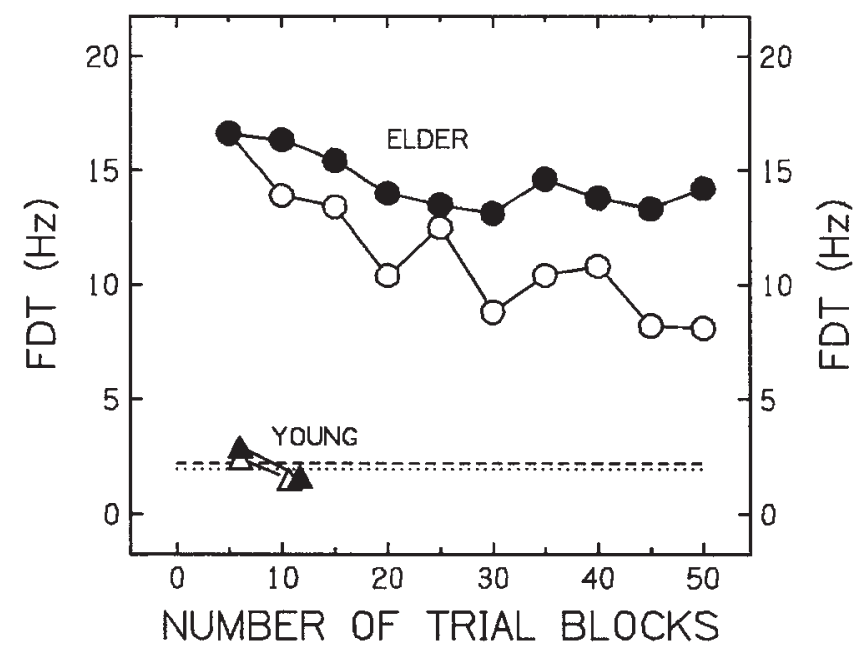

Fig. 2 For irrelevant level-difference conditions, the average FDTs of young (triangles) and older (circles) adults are shown as a function of training, expressed in terms of number of trial blocks. Open and solid symbols depict data for positive and negative irrelevant level differences, respectively. The horizontal lines (bottom) depict the average baseline FDTs of young (dotted line) and older adults (dashed line).

irrelevant level differences elevated the elders' FDTs to an average of $17 \mathrm{~Hz}$. Though the elevations tended to decrease with training, the elders showed FDTs of $8-14 \mathrm{~Hz}$ after completing an average of 50 trial blocks or $\approx 1,500$ trials on each polarity condition. The elders' FDT elevations were consistently lower with positive than with negative irrelevant level differences, but this trend did not reach statistical significance (see below).

In what follows, the terms initial and final FDT will designate the FDTs measured near the beginning and ending of the training period allotted to each irrelevant-difference condition. For each polarity condition, repeated-measures ANOVA were performed on the initial and final FDTs of both groups. On each ANOVA, the factors were age group and irrelevant difference, with 2 and 3 layers, respectively (the term "layer" is used here to avoid confusion with "level" difference). The layers of the irrelevant-difference factor were initial and final FDT, plus the baseline FDT measured in conditions without irrelevant differences. That is, the baseline FDT was treated as a layer in which the irrelevant difference was equal to zero. Paired comparison of baseline and initial FDTs assessed the significance of irrelevantdifference effects. The significance of training effects was assessed by paired comparison of initial and final FDTs, and by the Spearman-rank correlation between FDTs and training (i.e., number of trial blocks).

For positive irrelevant-difference conditions, the ANOVA revealed significant main effects of irrelevant difference $[F(2,20)=13.7, p<0.0002]$ and age $[F(1,20)=11.61, p<$ $0.0067]$. The interaction between age and irrelevant difference was significant also $[F(2,20)=11.69, p<0.0004]$, meaning that only the elders showed a significant irrelevant-difference effect. In older adults, Student-Newman-Keuls (SNK) tests reached significance with paired comparisons of baseline and initial, initial and final, but not of baseline and final FDTs; that is, the significant elevations in the initial FDTs were lowered by the significant training effect. In agreement with the ANOVA, the Spearman-rank correlation between FDTs and training was significant in all older adults. In young adults, neither the SNK paired-comparison tests nor the Spearmanrank correlation reached significance, because the irrelevant differences failed to elevate the baseline FDTs.

For negative polarity conditions, the ANOVA yielded trends similar to those with positive polarity. There were significant main effects of irrelevant difference $[F(2,20)=$ $10.11, p<0.0009]$ and age $[F(1,20)=9.47, p<0.0117]$, and a significant interaction between age and irrelevant difference $[F(2,20)=8.71, p<0.0019]$. For older adults, SNK tests reached significance with paired comparison of baseline and initial, baseline and final, but not of initial and final FDTs. The SNK test for training effects did not reach significance; however, the Spearman-rank correlation between FDTs and training was significant in all but one subject. This discrepancy occurs because the SNK test takes into account only the initial and final FDT, while the Spearman-rank correlation takes into account the entire set of FDTs collected over the training period. For young adults, neither the pairedcomparison tests nor the Spearman-rank correlation reached significance because, across conditions, the FDTs were very similar.

The significance of differences between polarity conditions was assessed with separate three-factor, repeatedmeasures ANOVA. The factors were age group, irrelevantdifference polarity, and training, with 2 layers each. Rather than the actual FDT values, the data for this ANOVA consisted of FDT differences between the baseline FDT and the initial or final irrelevant-difference FDTs. The ANOVA yielded significant main effects of age $[F(1,10)=10.98, p<0.0078]$ and training $[F(1,10)=20.38, p<0.0011]$, but not of irrelevant-difference polarity $[F(1,10)=1.78, p<0.212]$.

\section{Discussion and conclusions}

Irrelevant fluctuation in signal level elevated the FDTs of older but not of young adults; the tenfold elevations reported here are much larger than the tripling reported for young adults in previous studies [6,7]. Positive and negative irrelevant level differences yielded roughly the same effects in both age groups, though the elders' FDTs tended to be lower in the positive than in the negative polarity condition. While young adults are able to focus attention on target frequency increments and ignore irrelevant level differences, older adults have difficulty doing so. The FDT elevations induced by irrelevant level fluctuation suggest that interactions between pitch and loudness are stronger in older than in young adults; their ability to separate these dimensions at the perceptual level is reduced. Because the FDT elevations decreased with training, the ability to ignore irrelevant stimulus differences seems to depend also on prior knowledge about stimulus characteristics and long-term memory.

Peripheral-receptor injury cannot account for the elders' FDT elevations because their hearing sensitivity was normal; in addition, in the baseline condition, both age groups achieved virtually the same FDTs. This suggests that the 
inability to ignore irrelevant differences is linked to centralprocessing constraints, such as memory capacity and selective attention. In the baseline condition, discerning frequency increments may depend on within-trial comparisons of $T$ memory traces activated on each observation interval. In level-fluctuation conditions, the irrelevant level differences could be either 5 or $10 \mathrm{~dB}$, and have negative or positive polarity. Thus, relative to baseline, the level-fluctuation conditions conveyed 1.0 extra bit of information, computed as the $\log _{2}$ of the number of irrelevant-difference values (i.e., $\log _{2} 2$ ). Because the extra stimulus information increases the memory-capacity demands, one could assume that age effects reflect memory deficits. In agreement with the present results, this hypothesis does not predict polarity-dependent age effects because the increase in stimulus information is roughly equal for both polarities. The hypothesis is consistent also with the claim that working-memory capacity may be reduced in older adults $[1,8]$; irrelevant information draws on limited capacity working-memory resources, thereby degrading target-frequency discrimination.

On the other hand, on each irrelevant-difference trial, the target-frequency increments occurred in one of two stimulus contexts: in negative-polarity conditions, $T$ was softer in the comparisons than in the standard $T$; in positive-polarity conditions, $T$ was louder in the comparisons than in the standard. Therefore, irrelevant differences also established a context, which could have an impact on selective attention. Selective-attention deficits could account for limitations in the elders' ability to ignore irrelevant information [17,18].

Selective attention and memory capacity are closely intertwined, and extricating one from the other may not be possible. However, because the amount of stimulus information does not depend on polarity as the context effects do, the present results seem more in line with a memory-capacity than a selective-attention impairment: the elders' FDT elevations differed little across polarities, and keeping polarity constant throughout each trial block did not improve performance.

An attention-deficit account predicts polarity effects: since target frequency increments were positive in all cases, ignoring negative irrelevant-level differences was expected to be easier than the positive; the present trends are almost the opposite. Similarity between target and irrelevant information plays a major role in informational-masking paradigms [19]. In pitch-classification tasks [20], young adults perceive highpitched, loud sounds as being more congruent than highpitched, soft sounds; that is, pitch increments are perceived as been more similar to an increase than to a decrease in loudness. In pitch matching tasks with $40-\mathrm{ms}, 200-3200 \mathrm{~Hz}$ pure tones, pitch tends to decrease if the probe-tone level is higher than the standard, but it tends to increase if the level is lower than the standard [21]. Finally, in frequency discrimination of signals below $2.0 \mathrm{kHz}$, an increase in level tends to cancel out the pitch shift induced by a positive frequency increment, but a decrease in level tends to enhance the pitch shift [16]. The elders' weak polarity effects suggest that the main performance constraint is memory capacity rather than selective attention.

Improvements in the ability to ignore irrelevant information could be explained on the assumption that, with training, young adults develop long-term memory stimulus traces. Once established, these traces may allow listeners to anticipate what to listen for and what to ignore in the stimulus pattern, reduce the sensory- or working-memory load, and yield improved frequency discrimination [22].

\section{Acknowledgments}

The research was supported by a grant from the College of Allied Health, OU Health Sciences Center awarded to B. Espinoza-Varas (PI). The authors would like to thank the older and young adults for their willingness and enthusiasm to participate in this project and complete the test schedules.

\section{References}

[1] M. K. Pichora-Fuller, B. A. Schneider and M. Daneman, "How young and old adults listen to and remember speech in noise," J. Acoust. Soc. Am., 97, 593-608 (1995).

[2] S. Gordon-Salant and P. J. Fitzgibbons, "Recognition of multiply degraded speech by young and elderly listeners," J. Speech Hear. Res., 38, 1150-1156 (1995).

[3] P. A. Tun and A. Wingfield, "One voice too many: Adult age differences in language processing with different types of distracting sounds," J. Gerontol. Psychol. Sci., 54B, P317P327 (1999).

[4] D. E. Broadbent, Perception and Communication (Pergamon, London, 1958).

[5] N. Moray, Listening and Attention (Penguin Books, Baltimore, 1972).

[6] G. B. Henning, "Frequency discrimination of random-amplitude tones," J. Acoust. Soc. Am., 39, 336-339 (1966).

[7] N. Zagorski, "Perceptual independence of pitch \& loudness in a signal detection experiment: A processing model for 2ATFC (21FC) experiments," Percept. Psychophys., 17, 525-531 (1975).

[8] S. Gordon-Salant and P. J. Fitzgibbons, "Selective cognitive factors and speech recognition performance among young and elderly listeners," J. Speech Hear. Res., 40, 423-431 (1997).

[9] B. Espinoza-Varas and H. Jang, "Sequential interactions in the discrimination of frequency differences co-occurring with irrelevant differences in frequency, duration, or level," J. Acoust. Soc. Am., 102, 3161 (1997).

[10] B. Espinoza-Varas and H. Jang, "Sequential interactions in discrimination of target frequency differences trailed by irrelevant frequency differences: Effects of target duration and target-irrelevant frequency separation," in Proc. ICA1998, P. K. Kuhl and L. A. Crum, Eds., pp. 2343-2344 (1998).

[11] H. Levitt, "Transformed up-down methods in psychoacoustics," J. Acoust. Soc. Am., 49, 467-477 (1971).

[12] C. W. Turner, F. Zeng, E. M. Relkin and A. R. Horwitz, "Frequency discrimination in forward and backward masking," J. Acoust. Soc. Am., 92, 3102-3108 (1992).

[13] ANSI (American National Standards Institute), Specification for audiometers ANSI S3.6-1996 (American National Standards Institute, New York, 1996).

[14] E. Pfeiffer, "A short portable mental status questionnaire for the assessment of organic brain deficit in elderly patients," J. Am. Geriat. Soc., 23, 433-441 (1975).

[15] N. He, J. R. Dubno and J. H. Mills, "Frequency and intensity discrimination measured in a maximum-likelihood procedure from young and aged normal-hearing subjects," J. Acoust. Soc. Am., 103, 553-565 (1998).

[16] D. S. Emmerich, W. Ellermeier and B. Butensky, "A reexamination of the frequency discrimination of random- 
amplitude tones, and a test of Henning's modified energydetector model," J. Acoust. Soc. Am., 85, 1653-1659 (1989).

[17] L. Hasher and R. T. Zacks, "Working memory, comprehension, and aging: A review and a new view," in The Psychology of Learning and Motivation, G. H. Bower, Ed. (Academic Press, New York, 1988), Vol. 22, pp. 193-225.

[18] R. T. Zacks and L. Hasher, "Cognitive gerontolgy and attentional inhibition: A reply to Burke and McDowd," $J$. Gerontol. Psychol. Sci., 52B, P274-P283 (1997).

[19] D. L. Neff, "Signal properties that reduce masking by simultaneous, random-frequency maskers," J. Acoust. Soc. Am., 98, 1909-1920 (1995).

[20] R. D. Melara and L. E. Marks, "Perceptual primacy of dimensions: Support for a model of dimensional interaction," J. Exp. Psychol. Hum. Percept. Perform., 16, 398-414 (1990).

[21] T. D Rossing and A. J. Houtsma, "Effects of signal envelope on the pitch of short sinusoidal tones," J. Acoust. Soc. Am., 79, 1926-1933 (1986).

[22] R. Naatanen, Attention and Brain Function (Lawrence Erlbaum, Hillsdale, N.J., 1992). 